\title{
Goal-Conflict Detection based on Temporal Satisfiability Checking
}

\author{
Renzo Degiovanni ${ }^{*}$ Nicolas Ricci* ${ }^{*}$ Dalal Alrajeh ${ }^{\dagger}$ Pablo Castro* Nazareno Aguirre* \\ *Departamento de Computación, Universidad Nacional de Río Cuarto and CONICET, Argentina \\ †Department of Computing, Imperial College London, UK \\ \{rdegiovanni, nricci, pcastro, naguirre\}@dc.exa.unrc.edu.ar, dalal.alrajeh@ic.ac.uk
}

\begin{abstract}
Goal-oriented requirements engineering approaches propose capturing how a system should behave through the specification of high-level goals, from which requirements can then be systematically derived. Goals may however admit subtle situations that make them diverge, i.e., not be satisfiable as a whole under specific circumstances feasible within the domain, called boundary conditions. While previous work allows one to identify boundary conditions for conflicting goals written in LTL, it does so through a pattern-based approach, that supports a limited set of patterns, and only produces pre-determined formulations of boundary conditions.

We present a novel automated approach to compute boundary conditions for general classes of conflicting goals expressed in LTL, using a tableaux-based LTL satisfiability procedure. A tableau for an LTL formula is a finite representation of all its satisfying models, which we process to produce boundary conditions that violate the formula, indicating divergence situations. We show that our technique can automatically produce boundary conditions that are more general than those obtainable through existing previous pattern-based approaches, and can also generate boundary conditions for goals that are not captured by these patterns.
\end{abstract}

\section{CCS Concepts}

-Software and its engineering $\rightarrow$ Requirements analysis; Risk management; $\bullet$ Theory of computation $\rightarrow A u$ tomated reasoning; Modal and temporal logics;

\section{Keywords}

Goal Conflicts, Satisfiability Checking, Tableaux Method

\section{INTRODUCTION}

The derivation of correct software requirements specifications is essential to any reliable software development process [?]. With the ever increasing complexity of software, the importance of rigorous methods in supporting the attainment of correct specifications prior to their implementation, also increases. Much research over the last decades has demonstrated the significant advantages that formal, goal-oriented approaches bring to the generation of correct software requirements specification. Goals are prescriptive statements of how the system should behave. They reflect stakeholders' understanding of what the envisioned system is intended to do, and the criteria upon which it would be evaluated. They are commonly used to: aid the elicitation and elaboration of requirements [?, ?]; guide the refinement and organisation of requirements [?]; and support the derivation of software operations [?, ?]. However, for such tasks to be successfully achieved, the goals themselves must be correct, which is not often the case. Goals are typically too ideal to start with (wavering off the exceptional conditions that may arise within its environment once implemented [?, ?]), partial and imprecise. Ensuring their correctness within the development cycle is of utmost importance.

One of the challenges in specifying correct goals is ensuring their consistency. Inconsistency occurs when two or more goals cannot be satisfied simultaneously, owing to their contradictory nature, non-conformance to standards, or because of restrictions imposed within certain domains, amongst other reasons [?]. They are typically a result of overlapping and conflicting expressions. Detecting and resolving inconsistencies in goals (a process called inconsistency management) early on not only helps in avoiding costly software repairs but also supports systematic requirements' elicitation and verification activities [?, ?]. Several approaches have been proposed in the literature for managing inconsistency in goals. Much work has been done on the qualitative end, e.g., [?, ?], where the general focus has been on identifying contradictory low-level requirements and computing the degree to which goals are satisficed or denied by them. On the formal side, inconsistency management has also been the focus of several studies, e.g., [?, ?, ?, ?, ?].

A weaker notion of conflict (called divergence) in goals expressed in Linear Temporal Logic (LTL) [?] has been addressed in [?]. This latter type of inconsistency is concerned with those goals which are not contradictory (can be simultaneously satisfied), but become inconsistent when certain conditions hold. Consider for instance the following goals from the mine pump controller example [?]: "the pump shall be on when the water level is above the high threshold", and "the pump shall be off when methane is detected in the mine". These goals are not logically inconsistent as they are satisfiable in cases where the water level never reaches a high level or methane is not detected in the mine. They become logi- 
cally inconsistent only in the case when the water level is high and methane is present at the same time. Situations like the latter can be captured formally as assertions called boundary conditions, i.e., declarative formulas that characterise those particular circumstances that lead to inconsistency. Existing model synthesis approaches would not detect this type of inconsistency since there exists at least one model that satisfies such goals, in which the boundary condition never holds. So far, very limited work has been done on automatically finding boundary conditions for goal expressions, save for [?] which supposes goals expressed according to fixed templates written in LTL.

In this paper, we present a novel approach to automatically compute boundary conditions for conflicting goals expressed in LTL, using a satisfiability procedure based on tableaux. A tableau for an LTL formula is essentially a finite graph representation of all its satisfying models; it is built by first decomposing the formula whose satisfiability is being analysed, according to decomposition rules that produce, for temporal operators, constraints on the current state and future states, for their satisfaction. The resulting graph explores the possible ways of making the initial formula satisfiable, and in this process, contradictory portions are identified. The second phase of the tableau method removes contradictory portions, as well as parts of the graph that cannot satisfy eventualities, leaving a subgraph, the tableau, that captures all models of the formula (when it becomes empty, the formula is unsatisfiable). Intuitively, the tableau indirectly captures "conflicting situations", since any condition not included in the tableau necessarily prevents the formula from being satisfiable. Our approach consists of computing the tableau from a set $G$ of goals, and then exploring it to identify conditions that would "escape" the tableau, thus violating the goals, to produce boundary conditions. Our approach is general, in the sense that it can automatically detect conflicts in goals expressed as any LTL formula (as opposed to [?], where goals must comply with specific patterns for conflict detection). In particular, our approach can be applied to all patterns in [?]. In fact, as we will show, our technique produces more general boundary conditions than those of the pattern-based approach, while at the same time it allows us to compute boundary conditions from goals not captured by these patterns.

The rest of the paper is organised as follow. In Section ?? we present the basic concepts that will be necessary throughout the paper. In Section ?? we present an illustrating example, together with some observations that motivate the approach. The approach itself is described in detail, in Section ??. We then perform a validation of our technique (Section ??), by comparing it with the pattern-based mechanism for conflict detection, and by applying our approach on various case studies. Finally, we discuss related work in Section ??, and draw some conclusions and further work in Section ??.

\section{BACKGROUND}

\subsection{Goal-Oriented Modelling}

Goal-Oriented Requirements Engineering [?] proposes capturing how a system should behave through the specification of a set of high-level goals, that will drive the requirements engineering process. Goals are prescriptive statements that the envisioned system is expected to achieve through the cooperation of its agents (e.g., humans, devices and software) within a given domain. Domain properties are descriptive statements about the problem world (such as natural laws). In this setting, a goal model is a decomposition of goals through refinements, which essentially capture how a goal can be achieved in terms of simpler ones. These goal refinements end when each leaf subgoal can be assigned to a single agent. Agents provide operations, whose combined behaviours must fulfill the goals. A requirement is a terminal goal assigned to a software agent, while an assumption is a goal assigned to an agent in the environment.

In this context, inconsistent goals may arise from conflicting expressions. In particular, a weak form of conflict, called divergence, is of relevance in goal-oriented requirements engineering. A set of goals $G_{1}, \ldots, G_{n}$ is said to be divergent [?, ?] with respect to a set Dom of domain properties iff there exists a boundary condition $B C$ such that the following conditions hold:

$$
\begin{aligned}
& \left\{\text { Dom, BC, } \bigwedge_{1 \leq i \leq n} G_{i}\right\} \models \text { falser } \quad \text { (logical inconsistency) } \\
& \left\{\text { Dom, BC, } \bigwedge_{j \neq i} G_{j}\right\} \not \models \text { false, for each } 1 \leq i \leq n \text { (minimality) } \\
& B C \neq \neg\left(G_{1} \wedge \ldots \wedge G_{n}\right)
\end{aligned}
$$

Intuitively, conditions 1 and 2 indicate that the boundary condition captures a particular combination of circumstances that makes the goals conflicting. Roughly speaking, the first condition establishes that goals $G 1, \ldots, G_{n}$ cannot simultaneously be satisfied in Dom under any circumstances when $B C$ holds. The second condition states that removing any of the goals no longer results in a logical inconsistency. The third condition prohibits a boundary condition to be simply the negation of goals. Notice also that the minimality condition forbids trivial boundary conditions ( $B C$ cannot be false nor can it be the negation of one of the goals $\left(\neg G_{i}\right)$ ), and requires it to be consistent with the domain Dom.

\subsection{Linear-Time Temporal Logic}

Linear-Time Temporal Logic (LTL) is a formalism that has been extensively employed to state properties of reactive systems. LTL assumes a lineal topology of time, i.e., each instant is followed by a unique future instant, and its formulas are evaluated over infinite traces that represent system executions. Given a set $A P$ of propositional variables, LTL formulas are inductively defined using the standard logical connectives and temporal operators $\bigcirc$ and $\mathcal{U}$, as follows: (i) every $p \in A P$ is an LTL formula, and (ii) if $f_{1}$ and $f_{2}$ are LTL formulas, then so are $\neg f_{1}, f_{1} \vee f_{2}, f_{1} \wedge f_{2}, \bigcirc f_{1}$ and $f_{1} \mathcal{U} f_{2}$. We consider the usual definition for the operators $\square$ (always), $\diamond$ (eventually) and $\mathcal{W}$ in terms of $\bigcirc, \mathcal{U}$ and logical connectives. Temporal formulas are evaluated over infinite traces of propositional valuations. Formulas with no temporal operators are evaluated in the first valuation of a trace. Given a trace $\sigma, \bigcirc f$ is true in $\sigma$ iff $f$ is true in $\sigma[1 .$. (the trace obtained by removing the first valuation from $\sigma$ ), and $f_{1} \mathcal{U} f_{2}$ is true in $\sigma$ iff there exists a position $i$ such that $f_{2}$ holds in $\sigma[i .$.$] , and for all 0 \leq j<i, f_{1}$ holds in $\sigma[j .$.$] .$

In this work we focus on safety properties, typically specified as $\square f$, and a particular kind of liveness properties, namely those captured by the reachability and response patterns, specified as $\diamond f$ and $\square\left(f_{1} \rightarrow \diamond f_{2}\right)$, respectively. For further details on LTL and temporal patterns, see [?].

\subsection{The Tableau Method for LTL}


The tableau method is a well-known logical satisfiability approach, based on the decomposition of the formula being assessed according to the semantics of its logical operators, to search for satisfying valuations. In propositional logic, formulas are decomposed according to the semantics of boolean connectives: a model satisfies $f_{1} \wedge f_{2}$ iff it satisfies both conjuncts at the same time; a model satisfies $f_{1} \vee f_{2}$ iff it satisfies at least one of the disjuncts; and so on. Notice that if we start with the singleton containing the assessed formula and connect sets of formulas according to decomposition rules (e.g., $\left\{f_{1} \wedge f_{2}\right\}$ will be connected to $\left.\left\{f_{1}, f_{2}\right\}\right)$, then this tableau process leads to a finite tree, the tableau. Branches originate due to disjunction; finiteness is guaranteed because sets containing contradictions are not further expanded, and atomic formulas cannot be decomposed. In a propositional tableau structure, leaves not containing contradictions characterise sets of satisfying valuations: those obtained by assigning true (resp., false) to atomic variables appearing positively (resp., negatively) in the branch, and assigning any truth value to any other atomic proposition.

The tableau method for LTL extends propositional tableau with rules to cope with temporal operators. Temporal operators will lead to requirements on the "current" state in a trace, and on the rest of the trace. For instance, $\square f$ (globally $f$ ) will hold in a trace iff $f$ holds in the current state of the trace, and $\square f$ holds in the rest of the trace (or equivalently, if $\bigcirc \square f$ holds in the current state). On the other hand, $\diamond f$ leads to branching, as disjunction does: a trace satisfies $\diamond f$ iff the current state satisfies $f$ or the rest of the trace satisfies $\diamond f$ (or equivalently, $\bigcirc \diamond f$ is satisfied in the current state). Notice that these additional decomposition rules, if applied exhaustively, may lead to infinite branches. To avoid decomposing the same formula twice, decomposed formulas are marked, and if a decomposition leads to a previously constructed set, then the arc will lead to the already produced one (thus, LTL tableaux are graphs, as opposed to propositional tableaux, which are trees). Then, LTL tableaux will deal with sets of formulas and marked formulas (subformulas of that being assessed), connected according to decomposition rules. More precisely, LTL tableau works as follows. Initially, the tableau contains only one node, the root, consisting of the (unmarked) formula being queried for satisfiability. Then, each node will be decomposed according to the following rules:

$$
\begin{aligned}
& \begin{array}{cc}
\frac{f_{1} \wedge f_{2}}{f_{1}} \alpha & \square f_{1} \\
f_{2} & f_{1} \\
\square \square f_{1}
\end{array} \\
& \frac{f_{1} \vee f_{2}}{f_{1} \mid f_{2}} \beta \quad \frac{\diamond f_{1}}{f_{1} \mid \bigcirc \diamond f_{1}} \beta \quad \frac{f_{1} \mathcal{U} f_{2}}{f_{2} \mid f_{1} \wedge \bigcirc\left(f_{1} \mathcal{U} f_{2}\right)} \beta
\end{aligned}
$$

Notice that we do not have rules for negation; we assume the original formula to be in negation normal form (NNF), where negations are pushed to be applied to atomic propositions. Rules labelled with $\alpha$ do not lead to branches, whereas those labelled with $\beta$ produce branches.

Definition 2.1 (Elementary Formula). We call literal to a propositional variable or its negation. A formula is elementary iff it is a literal, or a temporal formula whose main operator is $\bigcirc$.

Then, the above rules are applied iteratively, taking into account marked vs unmarked formulas, and elementary formulas, as follows:
1. If $n$ is a node labelled with a set $S$ of formulas containing at least one unmarked non-elementary formula $f$, then: (i) if $f$ is a formula $\alpha$, and $\alpha_{1}, \alpha_{2}$ are the formulas resulting from decomposing $f$, then we create a node $n^{\prime}$ labelled with the set $(S-\{f\}) \cup\left\{\alpha_{1}, \alpha_{2}\right\} \cup\left\{f^{*}\right\}$, and then we connect $n$ with $n^{\prime}$; (ii) if $f$ is a formula $\beta$, and $\beta_{1}, \beta_{2}$ are the formulas resulting from decomposing $f$, then we create two nodes $n_{1}$ and $n_{2}$, such that, $n_{1}$ is labelled with the set $(S-\{f\}) \cup\left\{f^{*}\right\} \cup\left\{\beta_{1}\right\}$ and $n_{2}$ is labelled with $(S-\{f\}) \cup\left\{f^{*}\right\} \cup\left\{\beta_{2}\right\}$, and finally we connect $n$ with $n_{1}$ and $n_{2}$.

2. If $n$ is a node labelled with a set $S$ that contains only elementary and marked formulas, then we create a node $n^{\prime}$ labelled with the set $S^{\prime}$, such that, $\phi \in S^{\prime}$ iff $\bigcirc \phi \in$ $S$, and then we connect $n$ with $n^{\prime}$.

Definition 2.2 (States And Pre-States). The set of nodes $S_{N} \subset N$ that contain only elementary or marked formulas are called states, whereas the set $P_{N} \subset N$ that contains the initial root node and immediate successors of states are called pre-states.

After constructing the tableau, to decide if $\varphi$ is satisfiable, the unsatisfiable nodes must be eliminated from the graph. To do so, the following deletion rules are repeatedly applied:

1. If a node contains both a proposition $p$ and its negation $\neg p$, it is eliminated.

2. If all successors of a node have been eliminated, it is eliminated.

3. If a node is a pre-state that contains some eventuality $\diamond f_{2}$ or $f_{1} \mathcal{U} f_{2}$ that is not satisfiable, then it is eliminated. A formula $\diamond f_{2}$ or $f_{1} \mathcal{U} f_{2}$ is satisfiable in a pre-state iff there exists a path in the tableau leading from that pre-state to a node that contains formula $f_{2}$.

Intuitively, eventualities express "promises" that a property will eventually be fulfilled, so one must guarantee that a future state satisfies them, to consider these satisfiable.

The decision procedure ends when all unsatisfiable nodes have been removed from the tableau. If the initial node has been eliminated, the initial formula is unsatisfiable; if not, it is satisfiable. The reader is referred to [?] for further details and examples regarding the tableau construction for LTL.

As an example, consider the tableau in Figure ??, generated for the formula $\square \bigcirc p \wedge \diamond \neg p$. The root node contains $\{\square \bigcirc p, \diamond \neg p\}$ (we already decomposed the conjunction for space reasons). All nodes whose identifiers are in boldface $(n 2, n 3, n 5, n 8$ and $n 9)$ are states. Node $n 8$ cannot be further expanded since it contains a propositional inconsistency ( $p$ and $\neg p$ ). Notice also that all nodes reachable from $n 3$ contain $p$, and therefore they cannot fulfil the eventuality $\neg p$ associated to formula $\diamond \neg p$. The parts of the tableau that are eliminated according to the above rules are highlighted in the figure. Since root belongs to the tableau after all deletion rules have been applied, $\square \bigcirc p \wedge \diamond \neg p$ is satisfiable; the tableau also indicates the only way to make it satisfiable is in a trace in which the eventuality $\neg p$ is fulfilled in the first state, while in the rest of the trace $p$ is satisfied.

\section{MOTIVATING EXAMPLE}

To illustrate both the problem addressed and our proposed solution, let us consider a simplified version of the Mine Pump Controller (MPC) [?]. The MPC has a sensor that detects 


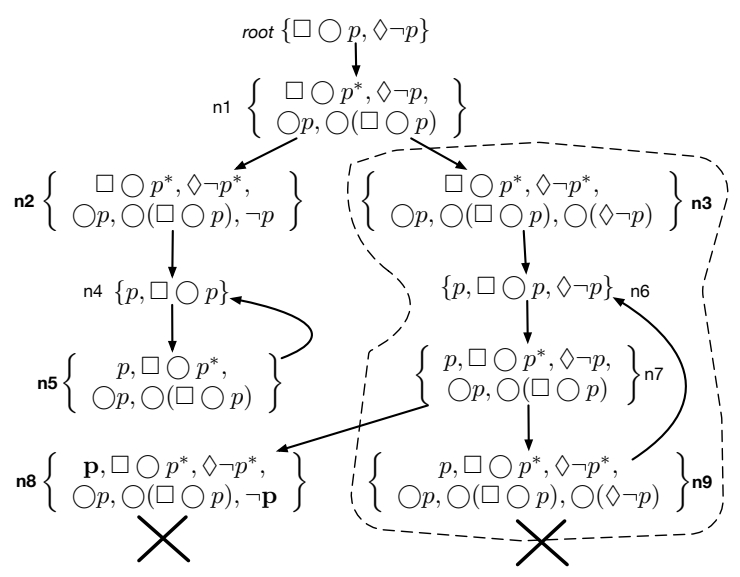

Figure 1: Tableau Example.

when the water level is high, and a sensor to detect the presence of methane in the environment. The propositional variables $h w, m$ and $p o$ are employed to represent the facts that a high water level is reached, that methane is present in the environment, and that the pump is on, respectively. In this context, the following goals are relevant:

Goal: Maintain[PumpOffWhenMethane]

InformalDef: The pump should be off when methane is detected in the mine.

FormalDef: $\square(m \rightarrow \bigcirc(\neg p o))$

Goal: Maintain[PumpOnWhenHighWater]

InformalDef: The pump should be on when the water level is above the high threshold.

FormalDef: $\square(h w \rightarrow \bigcirc(p o))$

While these goals can be simultaneously satisfied (e.g., when the water level is never high or methane is never present in the environment), they become logically inconsistent when, at the same time, the water level is high and methane is present. Such conflicting situations, in this case characterised by the boundary condition $\diamond(h w \wedge m)$, are not obvious to identify, and are very important to detect. We propose automatically detecting such boundary conditions by constructing and processing a tableau from the goals' temporal formalisations. Let us provide some intuition on how the process works, in the particular case of safety goals.

From the above safety goals (that we denote here as $G_{1}$ and $G_{2}$ for space reasons), the tableau method previously introduced produces the tableau shown in Figure ??. The root node is the only pre-state, and nodes $n 0, n 1, n 2$ and $n 3$ are states. Also, since $n 3$ contains both $p$ and $\neg p$, it is not further expanded (will be removed from the tableau). The dashed lines in the figure indicate that target nodes are reached through intermediate nodes (that are neither states nor pre-states).

States in the tableau capture sets of valuations in particular instants of a trace, through the literals they contain. For instance, state $n 0$ characterises valuations (or points during a trace execution) in which there is no methane and water level is not high $(\neg m \wedge \neg h w)$. Moreover, if the "consistent" states in a tableau are identified (basically, the states of the tableau that remain after the removal phase), since these characterise all consistent situations, the negation of their disjunction indirectly captures how the goals would be violated (would

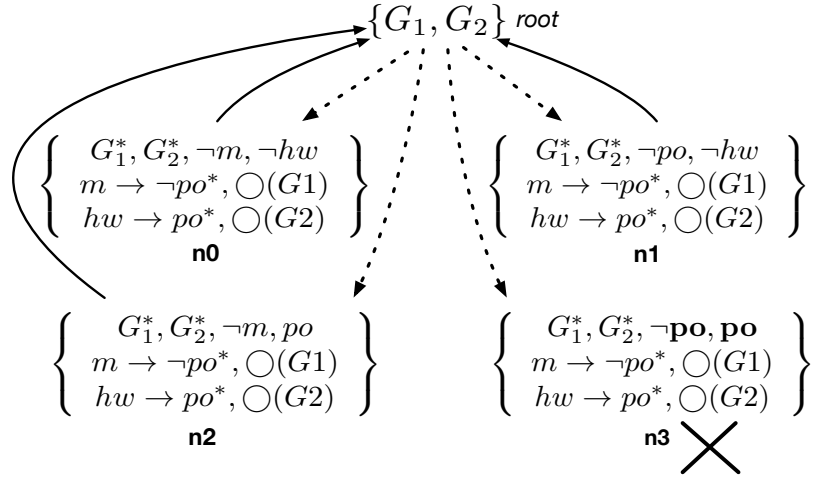

Figure 2: Tableau for the Mine Pump Controller. "fall" into the unsatisfiable part of the tableau). In our particular example, this formula is $\neg((\neg m \wedge \neg h w) \vee(\neg m \wedge$ $p o) \vee(\neg p o \wedge \neg h w))$, and reaching it leads to a violation of the goals. Thus, $\diamond \neg((\neg m \wedge \neg h w) \vee(\neg m \wedge p o) \vee(\neg p o \wedge \neg h w))$ is a boundary condition (or equivalently $\diamond((\neg h w \wedge m \wedge p o) \vee$ $(h w \wedge(m \vee \neg p o)))$, as our tool produces it after simplification), evidencing a weak conflict between the goals. Actually, as we will explain later on, this is in fact a potential boundary condition, since it may not satisfy minimality (an additional checking must be performed on our produced violations to guarantee minimality).

It is worth noting that our computed boundary condition is more general than that manually identified above $(\diamond(h w \wedge m))$. By more general we mean that the formula $\diamond(h w \wedge m)$ implies the boundary condition computed by our approach. As we will show in our experimental assessment, this tableaubased approach produces more general conflicts than those obtained using alternative pattern-based techniques. In the next section, we describe in detail the approach, including how other kinds of goals (besides safety) are handled.

\section{THE APPROACH}

Our tableau-based approach to automatically detect goal conflicts receives a goal-oriented requirements specification composed of LTL formulas capturing the domain assumptions Dom, as well as goals $G=\left\{G_{1}, \ldots, G_{n}\right\}$. The process may determine that there are no conflicts, or that there exist either strong or weak conflicts. The most relevant case, upon which we concentrate in this work, is when weak conflicts are detected. If this is the case, our process produces a set $B C=\left\{B C_{1}, \ldots, B C_{k}\right\}$ of boundary conditions capturing different divergent situations between the goals in the domain.

Our approach is able to deal with safety goals and a particular kind of liveness goals, namely those that can be expressed following the reachability or response (progress) patterns [?]. Reachability goals have the form $\diamond f$, while response goals have the form $\square\left(f_{1} \rightarrow \diamond f_{2}\right)$. Progress goals, on the other hand, are expressed as $\square \diamond f_{2}$, and are a particular case of response goals (those where $f_{1}=$ true) [?].

The overall approach is summarised in the following steps. (1) Tableau generation and refinement: A tableau structure $T$ for $D o m \wedge G$ is automatically generated using the LTL decision procedure presented in Section ??. As mentioned before, structure $T$ encodes all models of $D o m \wedge G$. If after the deletion rules are applied all the nodes are removed from 


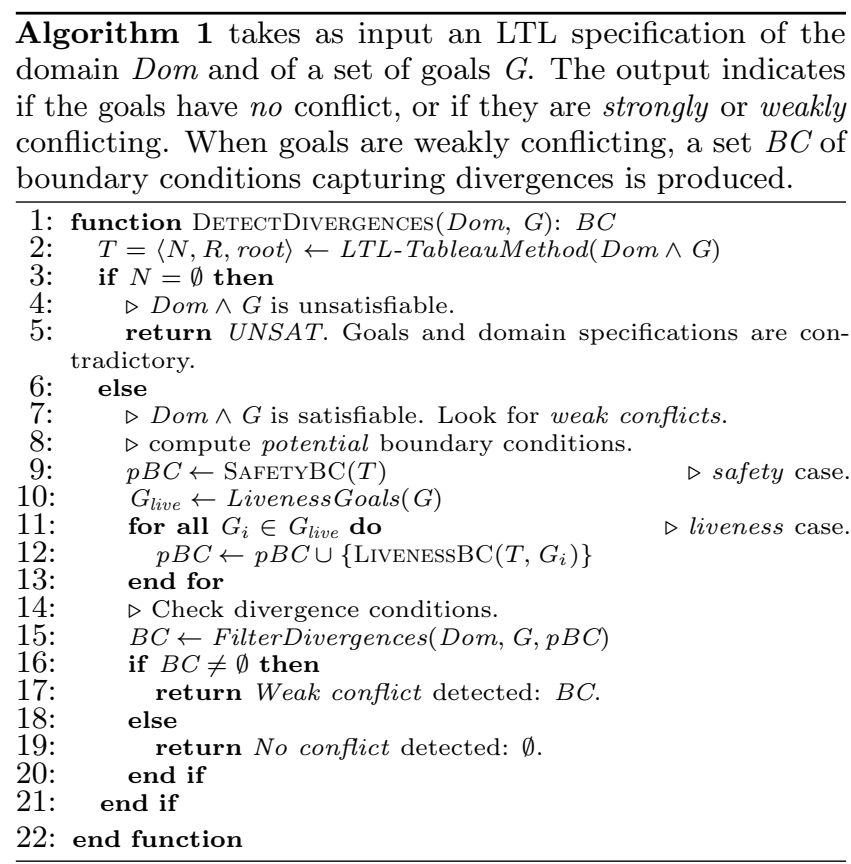

$T$, then goals and domain specifications are contradictory, and we do not look at it any further; otherwise, we proceed to look for weak conflicts.

(2) Potential $\boldsymbol{B} \boldsymbol{C}$ identification: At this point, tableau $T$ produced in the previous phase is non-empty, and characterises all the models for $\operatorname{Dom} \wedge G$, in the sense that every sequence that satisfies $\operatorname{Dom} \wedge G$ is a path in the tableau $T$, and every finite path obtained from $T$ is the prefix of some model of $D o m \wedge G$ [?]. However, there may exist some infinite paths in $T$ that leave some eventuality formula unsatisfied, and thus, such paths do not satisfy Dom $\wedge G$.

Taking these observations into account, from $T$, there are two potential sources of divergences that must be analysed. On one hand, all the unsatisfiable nodes removed by the application of the tableau deletion rules characterise a particular kind of violation to $\operatorname{Dom} \wedge G$. A condition that would force one to reach the inconsistent portion removed from $T$, may represent a divergence for Dom and $G$. We call this the safety case. On the other hand, given a liveness goal $G_{i} \in G$ whose eventuality is $f$ (e.g., a reachability goal $G_{i}=\diamond f$ ), an (infinite) path in the tableau $T$ that does not pass through a state in which $f$ holds, is a violation for $G_{i}$ and a potential source of divergence between the goals. We call this the liveness case. Why these cases are potential divergences and not actual divergences is further discussed later on, and analysed in the next phase. This current phase will produce a set of LTL formulas characterising potential divergences for both the safety and liveness cases.

This phase is achieved by extracting from tableau $T$ a set of path conditions that characterise the two kinds of inconsistencies just described. A path condition is an LTL formula characterising a path in the tableau (see Section ??). To produce path conditions, $T$ is traversed starting from the root node, collecting the information provided by the states to build LTL formulas that capture the following situations. For the safety case, the produced LTL formula captures a path that escapes from $T$, directing us into the unsatisfiable nodes (those removed from the tableau during node deletion). For the liveness case, the produced LTL formula characterises a path in $T$ that avoids the states in which the eventuality of some liveness goal is fulfilled.

Path conditions are used to produce the potential boundary conditions $p B C=\left\{p B C_{1}, \ldots, p B C_{m}\right\}$, whose concrete formulation depends on whether they are safety or liveness cases. As previously mentioned, formulas in $p B C$ are potential conflicts, because although they characterise violations for $\operatorname{Dom} \wedge G$, they do not necessarily represent a divergence case between the goals with respect to the domain. More precisely, according to the divergence definition given in Section ??, we have to check if the logical inconsistency and minimality conditions are satisfied, to guarantee that these are divergences.

(3) $\boldsymbol{B} \boldsymbol{C}$ extraction: This final phase consists of removing from $p B C$ those conditions that do not satisfy the divergence properties in Section ??. The remaining boundary conditions $B C=\left\{B C_{1}, \ldots, B C_{k}\right\}$ capture weak conflicts between the goals in $G$ and the domain Dom.

Algorithm ?? describes how the above steps are combined. We provide further details of each step below.

\subsection{Generating and Refining the Tableau}

LTL-TableauMethod (line 2 in Algorithm ??) implements the LTL decision procedure introduced in Section ??. It generates a tableau structure $T=\langle N, R$, root $\rangle$, which encodes all potential models for Dom $\wedge$ G. Recall that the formula to which the tableau construction is applied (in our case Dom $\wedge G$ ) is satisfiable iff the root node is not removed during the application of the deletion rules. Notice that in Algorithm ?? we simply indicate that the goals and domain constraints are contradictory, when the tableau is empty (root node removed). When, on the other hand, $T$ maintains some nodes after the application of the deletion rules, then Dom $\wedge G$ is satisfiable. In this case, the approach proceeds to generate potential boundary conditions, as described below.

\subsection{Generating Potential Boundary Conditions}

After generating the tableau $T$, the approach proceeds to extract from $T$ a set of of LTL formulas that characterise potential boundary conditions. More precisely, functions SAFETyBC and LivenEssBC in Algorithm ?? generate these LTL formulas for the safety and liveness cases, respectively. In both cases, the LTL formulas are generated in two steps. First, SAFETyBC computes a set of frontier path conditions that escape from $T$ directing us into the unsatisfiable nodes, previously removed by the tableau method. Similarly, LiveNESSBC first computes a set of path conditions in $T$ that avoid passing through the states in which the eventuality of some liveness goal is fulfilled. Then, given these path conditions, SAFETyBC and LivenessBC generate the set of potential boundary conditions from these computed path conditions. In the remainder of this subsection, we introduce some terminology necessary to present the process for extracting potential boundary conditions from a tableau, and then we further describe the above mentioned functions.

\section{State Constraints, Successors and Path Conditions.}

Let $T=\langle N, R$, root $\rangle$ be a tableau structure. The state nodes of $T$ (see definition ??) contain literals that represent the constraints that the states should satisfy to be part of a model for the formula that was queried for satisfiability.

Definition 4.1 (State Constraints). Given a state node $s \in S_{N}$, the state constraints imposed by $s$, denoted by 
CONS(s), are composed of the conjunction of literal formulas that $s$ contains. Given a set $S \subseteq S_{N}$ of state nodes, $C O N S(S)$ is defined as $\bigvee_{s \in S} C O N S(s)$.

$C O N S(s)$ then returns a propositional formula that characterises all states that satisfy the conditions imposed by state $s$. For instance, if $s=\left\{\square(p \vee \neg q)^{*}, \diamond r^{*}, p \vee \neg q^{*}, \neg q, r\right\}$, then $C O N S(s)=\neg q \wedge r$, characterising the states in which the proposition $q$ is false and $r$ is true.

Notice that, according to definition ??, some nodes in $N$ are neither states nor pre-states. For the sake of simplicity, let us ignore all these intermediate nodes, preserving the transition relation between states and pre-states in $R$.

Definition 4.2 (SuCCESSORS). Given two pre-state nodes $d_{1}, d_{2} \in P_{N}, \operatorname{succs}\left(d_{1}, d_{2}\right)=\left\{s \in S_{N} \mid\left(d_{1}, s\right) \in R \wedge\right.$ $\left.\left(s, d_{2}\right) \in R\right\}$, i.e., the set of state nodes that transit from $d_{1}$ to $d_{2}$. We will use succs $(d)$ to denote all state nodes that are successors of pre-state $d$, i.e., $\operatorname{succs}(d)=\left\{s \in S_{N} \mid(d, s) \in R\right\}$.

Definition 4.3 (РАTh). A path $\rho=\left(d_{0}, S_{0}\right) ;\left(d_{1}, S_{1}\right)$;$\ldots ;\left(d_{k}, S_{k}\right)$ in $T$ is a sequence of pairs of a pre-state node and $a$ set of state nodes such that: 1. $d_{0}=$ root; 2. $\forall i: 0 \leq$ $i<k, S_{i} \subseteq \operatorname{succs}\left(d_{i}, d_{i+1}\right)$, and $S_{k} \subseteq \operatorname{succs}\left(d_{k}\right) ; 3 . \forall i: 0 \leq$ $i \leq k, S_{i} \neq \emptyset$.

$A$ loop-free path is a path where $d_{i} \neq d_{j}$, for $0 \leq i<j \leq k$.

Definition 4.4 (PATh Condition). A path condition $\left[\varphi_{0}, \ldots, \varphi_{k}\right]$ is a sequence of propositional formulas such that there exists a path $\rho=\left(d_{0}, S_{0}\right) ;\left(d_{1}, S_{1}\right) ; \ldots ;\left(d_{k}, S_{k}\right)$ for a given tableau $T$, where $\varphi_{i}=\operatorname{CONS}\left(S_{i}\right)$, for all $0 \leq i \leq k$.

Intuitively, a path and its corresponding path condition characterise consistent ways of traversing the tableau $T$. Our approach will consider loop-free paths to compute path conditions for producing potential boundary conditions. The frontier path condition defined below is used for describing the paths that "cross" the frontier of the consistent part of the tableau, and lead to an inconsistent portion of it.

Definition 4.5 (Frontier Path Condition). $A$ frontier path condition $\left[\varphi_{0}, \ldots, \varphi_{k-1}, \psi\right]$ is a sequence of propositional formulas such that there exists in $T$ a path condition $\rho=\left[\varphi_{0}, \ldots, \varphi_{k}\right]$, and $\psi=\neg \varphi_{k}$.

\subsubsection{Potential Safety Boundary Conditions}

Function SAFETYBC given in Algorithm ?? receives as input the tableau structure $T=\langle N, R$, root $\rangle$ and returns a set of potential boundary conditions. First, it calls function SAFETyFPCs (line 3) to compute a set of frontier path conditions, capturing paths that "escape" from $T$ reaching the unsatisfiable nodes removed during the tableau construction. SAFETYFPCs explores $T$ starting from the root node (invocation in line 3 ), and traverses $T$ using the successors definition given above (set $S_{i}$ in lines 19-21), recovering path conditions (line 22) required to finally reach the inconsistent portion of the tableau (set $S_{k}$ and formula $\psi$ in lines 14-15). The algorithm keeps track of the already visited pre-states nodes, so loops are not considered (lines 11-13). Intuitively, SAFETYFPCs computes all the shortest loop-free frontier path conditions in $T$, that start in the root node and escape from $T$, ending in the unsatisfiable portion removed previously from $T$. Then, function SAFETYBC encodes each frontier path condition as an LTL formula $f$, by nesting each condition in the path using the next temporal operator $\bigcirc$ (line 5). The resulting formula $f$ is used to generate a potential boundary condition $\diamond(f)$ (line 6$)$. Intuitively, this formula indicates that there might be a conflict in our model if condition $f$ is eventually reached, since it would lead to reaching the inconsistent part of the tableau. Notice that by applying $\diamond$ to $f$ we are somehow "generalising" the inconsistency case $f$. This is necessary because otherwise, and due to our analysis being limited to loop-free paths, the obtained divergences would be too strong (representing divergent cases of a fixed length). This generalisation step may however make the candidate boundary condition consistent with the goals and domain, so we will have to later on check for inconsistency with respect to these constraints (see Section ??).

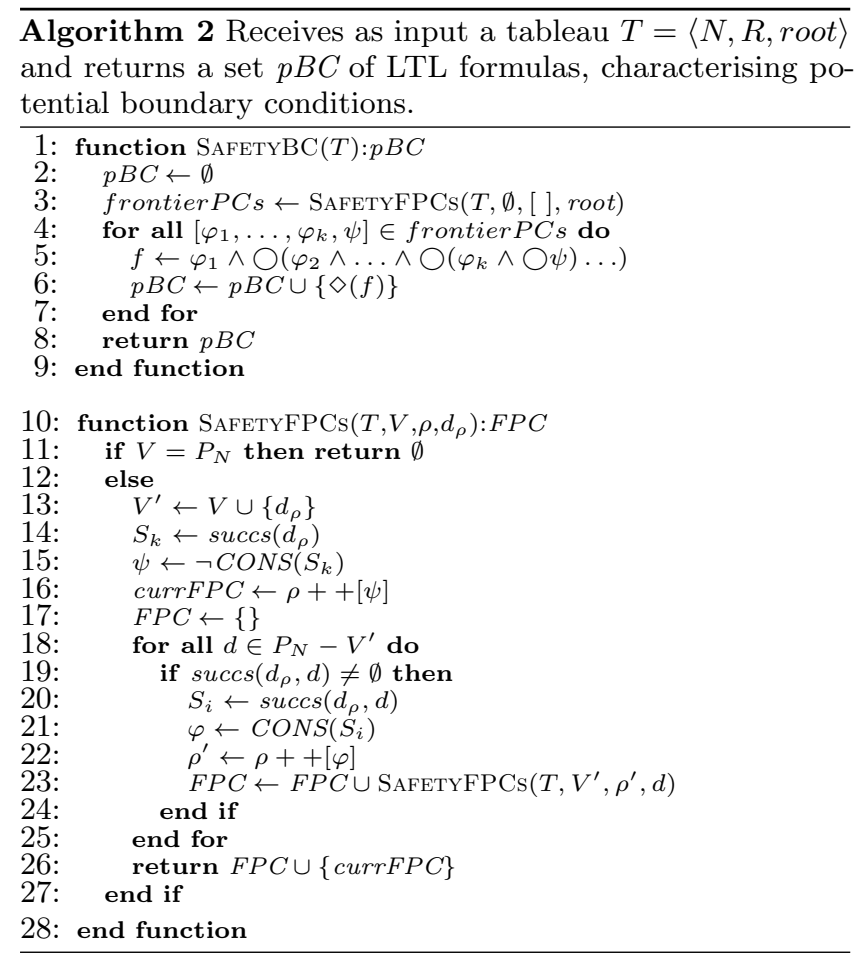

\subsubsection{Potential Liveness Boundary Conditions}

Function LivenEssBC in Algorithm ?? takes as input the tableau $T$ and a liveness goal $G_{i} \in G_{\text {live }}$, and returns an LTL formula that characterises different paths in $T$ that do not guarantee the eventuality corresponding to the goal $G_{i}$. This LTL formula represents a potential divergence between goal $G_{i}$ and the other goals and domain constraints. LiVENESsBC starts by identifying the nodes in $T$ in which the eventuality required by $G_{i}$ is fulfilled. That is, if $G_{i}$ is a reachability goal $\diamond f$ or a response goal $\square(g \rightarrow \diamond f)$, then $\diamond f$ is the eventuality that $G_{i}$ should fulfil. Then, sentence $E \leftarrow$ Eventualities $\left(T, G_{i}\right)$ in line 2 will store in $E$ the set of all nodes from $T$ that contain formula $f$ (i.e., each node in $E$ satisfies the eventuality demanded by goal $G_{i}$ ). For instance, if $G_{i}=\diamond p$, then all nodes from $T$ that contain proposition $p$, will be in the set $E$.

After that, LIVENESSFPCs computes all the loop-free path conditions in a way similar to that for the safety case. However, for the liveness case, the path conditions computed avoid those paths that pass through the nodes in $E$. Then, 
the main difference with the safety case is the composition of the sets $S_{i}$ and $S_{k}$ in Algorithm ??. In particular, set $S_{i}$ only considers state nodes that transition to the next pre-state node, but that are not included in the set $E$ (line 21). The set $S_{k}$ only contains the nodes that are in $E$ (line 18), thus $\psi$ (i.e., $\neg \operatorname{CONS}\left(S_{k}\right) \wedge \operatorname{CONS}\left(\operatorname{succs}\left(d_{\rho}\right)-E\right)$ ) characterises the nodes that do not satisfy the eventuality (line 19).

LIVENESSFPCs then returns the shortest loop-free path conditions that avoid reaching nodes in which the eventuality required by $G_{i}$ holds. In line 6 , a formula $f$ characterising each path condition, is generated. The disjunction of all these formulas captures all the different ways of traversing the tableau without hitting a state in which the eventuality of goal $G_{i}$ is satisfied (line 7 ). If the goal is a reachability one, of the form $G_{i}=\diamond f$, and the computed path condition is $F C$, then $\square(F C)$ is the potential divergence (line 12). This LTL formula indicates that there is a conflict if the condition $F C$ always holds, preventing the eventuality $f$ required by $G_{i}$ from being fulfilled. If, on the other hand, the goal is a response one, of the form $G_{i}=\square\left(f_{1} \rightarrow \diamond f_{2}\right)$, and the computed path condition is $F C$, then $\diamond\left(f_{1} \wedge \square(F C)\right)$ is the potential divergence (line 10). This LTL formula indicates that there is a conflict if $f_{1}$ holds at some point, but the eventuality $f_{2}$ is never fulfilled. Notice that $f_{2}$ may be satisfied before the condition $f_{1}$ holds, but it will not be satisfied an infinite number of times. The case of a progress goal $\square \diamond f_{2}$ is the same as for response, simply taking $f_{1}=$ true. Notice again that, in both cases, by putting $F C$ in the scope of $\square$ we are generalising the divergent case. Intuitively, formula $\square F C$ tries to characterise the infinite paths in the tableau that do not satisfy the $G_{i}$ 's eventuality.

Despite the fact that the generated LTL formulas capture conflicts with $\operatorname{Dom} \wedge G$, these may be discarded in the following phase because they may not meet all the requirements to be considered divergences, e.g., the minimality condition.

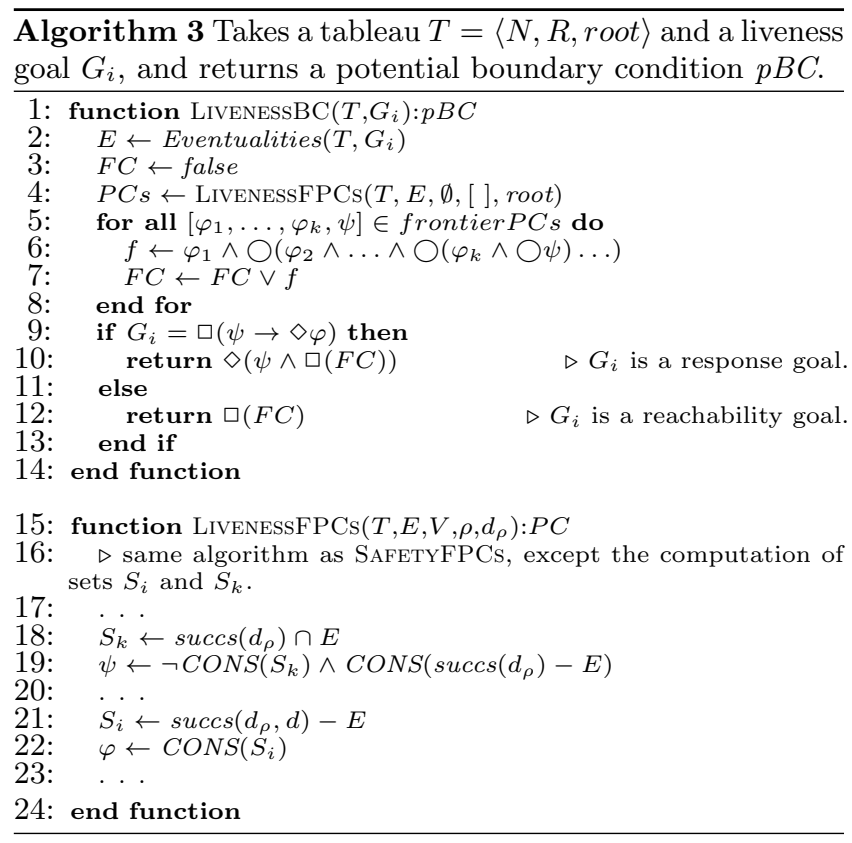

\subsection{Filtering Boundary Conditions}

Continuing with Algorithm ??, after computing the set $p B C$ of potential boundary conditions for the safety and liveness cases, function FilterDivergences (Dom, $G, p B C)$ is concerned with checking which ones represent actual divergences. The previous phase produces potential boundary conditions $p B C$, that may fail to be actual boundary conditions because of various reasons: (i) they might be consistent with the goals and domain constraints; (ii) they might not be minimal; or (iii) they might be the negation of a goal. Situation (i) can arise because, despite the fact that we compute path conditions that indeed contradict the goals and domain specification, since such path conditions are loop-free we "generalise" them (weaken them) by applying a temporal operator; such generalisation may make some potential boundary conditions consistent with the goals and domain constraints. Situations (ii) and (iii) are more clear: these impose conditions on our potential boundary conditions that the process to generate them does not take into account, and thus they must be checked afterwards. We then have to go through an additional process, represented by function FilterDivergences that for each LTL formula $b c \in p B C$, checks whether $b c$ meets the conditions described in Section ??, that define divergences (i.e., inconsistency with goals and domain specification, minimality with respect to the set $G$ of goals, and that $b c$ is not a trivial boundary condition). FilterDivergences checks inconsistency with goals and domain specification by assessing the satisfiability of $b c \wedge G \wedge D o m$ using an LTL satisfiability procedure; minimality, i.e., that for each goal $G_{i} \in G$, Dom $\wedge \bigwedge_{j \neq i} G_{j} \wedge b c$ is satisfiable, is also checked using LTL satisfiability; finally, checking that $b c \neq \neg G$ is just a syntactical check. FilterDivergences discards those LTL formulas in $p B C$ that do not meet the above conditions, and returns a set $B C \subseteq p B C$ of boundary conditions for the goals $G$ in the domain Dom.

FilterDivergences needs, for each potential boundary condition, to perform as many calls to the LTL decision procedure as goals are in $G$ (to check the minimality condition). To efficiently perform all these checks, we use Aalta [?], an efficient LTL satisfiability checker, recently developed. The experimental evaluation in Section ?? shows that the time required by FilterDivergences is small, compared to the time required to build the tableau.

\subsection{Correctness and (In)completeness}

Let us discuss now termination, correctness and (in)completeness of our approach. Regarding termination, recall that in Section ?? we explained that the tableau generation process is guaranteed to terminate, and the structure generated is a finite graph. So, the tableau contains a finite number of pre-state and state nodes. Since the functions to compute the path conditions, SAFETyFPCs and LivenEssFPCs, consider only loop-free paths over a finite graph, these are finitely many, and thus SAFETYFPCs and LivenEssFPCs terminate. All the checks performed in the last phase of the approach, FilterDivergences, are made using the LTL satisfiability checker Aalta [?] (that is guaranteed to terminate), for a finite number of potential boundary conditions. Thus, the whole process is guaranteed to terminate.

Regarding the correctness of the approach, i.e., that if the process produces a formula $b c$ this is indeed a boundary condition, notice that the last phase of our approach, FilterDivergences, checks that $b c$ satisfies all the conditions of the definition of boundary condition (see Section ??), thus guaranteeing correctness. 
Regarding completeness, i.e., that if there exists a divergence situation between the goals and the domain our process is able to produce it, the situation is different. Unfortunately our approach is not complete. Let us provide an example. Consider again goals $\square \bigcirc p$ and $\diamond \neg p$, used in Section ?? to illustrate a tableau. As we mentioned, these goals can be satisfied only when the eventuality $\neg p$ is fulfilled in the first state, and in the rest of the trace $p$ holds. So, if $p$ holds in the initial state, the goals diverge. In fact, $p$ is indeed a boundary condition, it meets the three properties defining it in Section ??. However, our approach cannot compute it, since we only produce formulas that contain at least one temporal operator. In fact, our approach will produce formulas $\square p, \diamond p$ and $\diamond(\neg p \wedge \bigcirc \neg p)$ as potential boundary conditions, none of which satisfy the boundary condition definition and thus are removed by FilterDivergences.

\section{EVALUATION}

In this section we evaluate our proposal, addressing the following research questions:

RQ1 Is our approach well suited to detect divergences in goal specifications?

RQ2 Are the boundary conditions computed by our approach more general than those derived by related techniques?

RQ3 Does our approach apply to specifications that cannot be handled by related techniques?

To answer RQ1, we take various case studies from the literature on formal requirements specifications, that feature both safety and liveness goals, and evaluate our technique for computing divergences. Section ?? reports the results of analysing two case studies: the Elevator Controller [?] and the Rail Road Crossing System [?]. To answer RQ2, in Section ?? we briefly introduce the pattern-based approach to goal conflict detection from [?], and compare divergences computed using our approach against those obtained using the pattern-based one. To answer RQ3, in Section ?? we study a simplified version of the TCP protocol, whose goals do not correspond to any of the patterns of the previous approach from [?], to assess how our approach deals with them. Finally, in Section ?? we provide further examples, and discuss about the scalability of our approach and the succinctness of the computed boundary conditions.

These research questions are answered using a tool that we developed, that implements our tableau based goal conflict detection approach. The tableau generation and potential boundary conditions computation is implemented in Haskell, and employs a BDD library [?] to simplify expressions when path conditions are collected, while computing boundary conditions. Moreover, the tool integrates the LTL satisfiability checker Aalta [?], to efficiently perform all the SAT checks required by the FilterDivergences phase.

The tool, the specifications for all case studies, and a description of how to reproduce the experiments can be found in http://dc.exa.unrc.edu.ar/staff/rdegiovanni/ase2016. All the experiments were run on an Intel Core i5 4460 processor, 3.2Ghz, with $8 \mathrm{~Gb}$ of RAM, running GNU/Linux (Ubuntu 15.04). For each case study we report the size of the constructed tableau (number of nodes and transitions), the number of computed potential boundary conditions, the number of resulting divergences, and the analysis time required for constructing the tableau and for the entire process. This information is summarised in Section ??.

\subsection{Case Studies}

We evaluate two case studies taken from the literature, the Elevator Controller introduced in [?], and a simplified version of the Rail Road Crossing System taken from [?].

\subsubsection{Elevator Controller}

The Elevator Controller [?] has one sensor call to detect when a user has called the elevator, and one sensor atfloor that is set to true when the elevator reaches the floor where the user is waiting. In addition, the controller sends an open signal indicating the door must open. For simplification, we assume there is only one user that may call the lift at a time. The following goal and domain properties are elicited:

Goal: Achieve[Open When Call]

FormalDef: $\square($ call $\rightarrow \diamond($ open $))$

Domain: Maintain[DoorOpensWhenAtFloor]

FormalDef: $\square(\bigcirc($ open $) \rightarrow$ atfloor $)$

The goal Open WhenCall indicates that the controller should respond to the user's calling by opening the door. The domain property DoorOpens WhenAtFloor captures the door's behaviour, indicating the door opens only when the elevator reaches the floor where the user is waiting. Our approach computes the following 2 potential boundary conditions:

$$
\begin{aligned}
& \text { 1. } \diamond(\text { call } \wedge \neg \text { atfloor } \wedge \neg \text { open } \wedge \bigcirc(\text { open })) \\
& \text { 2. } \diamond(\text { call } \wedge \square(\neg \text { open } \vee(\text { call } \wedge \neg \text { atfloor } \wedge \text { fopen } \wedge \bigcirc(\neg \text { open }))))
\end{aligned}
$$

Condition 1 is computed for the safety case, while condition 2 is for the liveness goal. Only condition 2 meets the definition of divergence (condition 1 is inconsistent with the domain because the door will open in the next state, but the elevator is not at the floor where the user called it). This formula captures the scenarios in which the elevator has been called but the elevator will not be at the floor where it was called, and it will never open the door. The whole process to compute this boundary condition takes 0.80 seconds.

\subsubsection{The Rail Road Crossing System}

Consider now a simplified Rail Road Crossing System [?]. In this model, a train can approach and enter a crossing, captured by $t a$ and $t c$, respectively. A car may also approach and enter the crossing, captured by $c a$ and $c c$, respectively. The crossing gate may be opened ( go) or closed $(\neg g o)$. The following goals and domain properties are elicited:

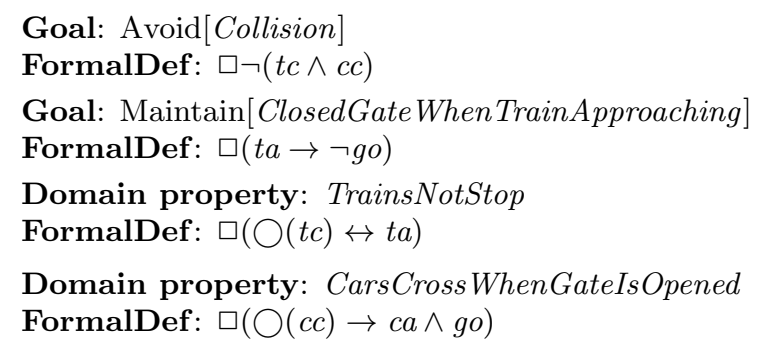

Our tool computes 5 potential boundary conditions, with only one being a divergence, in 0.5 seconds. The identified divergence is the following:

$$
\text { 1. } \diamond((\neg c c \wedge g o \wedge t a) \vee(c c \wedge(\neg g o \wedge t c \vee g o \wedge(t a \vee t c))))
$$

This boundary condition reveals a few dangerous situations. A conflict arises if the gate is open when the train is approaching, and the car has not crossed yet. Other similar conflicting situation arise when the car is crossing at the same time as the train is approaching or crossing. 


\subsection{Comparison with Divergence Patterns}

We now compare our technique with the only previous formal approach to derive boundary conditions, presented in [?]. This previous approach requires matching goals against a set of pre-defined divergence patterns, for which divergence expressions are provided. To answer RQ2, we compare these divergences with those computed by our approach, when fed with the pre-defined patterns from [?]. Figure ?? summarises the three divergence patterns presented in [?], in which the goals and domain are specified in LTL.

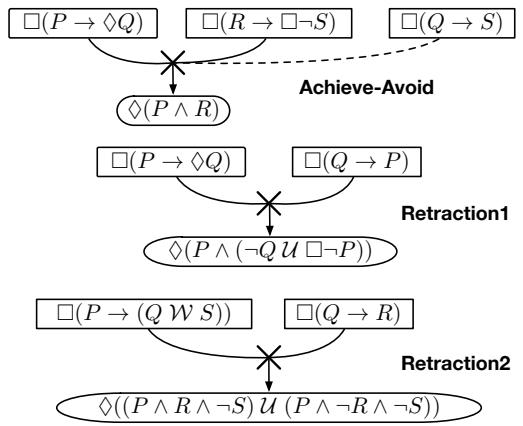

Figure 3: Divergence Patterns from [?].

In the case of the Achieve-Avoid pattern, the following potential boundary conditions are produced, resulting all in divergences:

$$
\begin{aligned}
& \text { 1. } \diamond(\neg P \wedge(\neg Q \wedge R \wedge S \vee Q \wedge(R \vee \neg S)) \\
&\vee P \wedge(\neg Q \wedge R \vee Q \wedge(R \vee \neg S))) \\
& \text { 2. } \diamond(\neg P\wedge \neg Q \wedge R \wedge \neg S \wedge \bigcirc(P \vee Q \vee S)) \\
& \text { 3. } \diamond(P \wedge \neg Q \wedge \neg R \wedge \bigcirc(\neg Q \wedge R \vee Q \wedge(R \vee \neg S))) \\
& \text { 4. } \diamond(P \wedge \square((P \wedge \neg Q \wedge \neg R) \vee \\
& \quad(\neg P \wedge \neg Q \wedge(\neg R \vee \neg S)) \vee \\
& \quad(P \wedge \neg Q \wedge \neg R \wedge \bigcirc(\neg Q \wedge \neg R)) \vee \\
&\quad(\neg P \wedge \neg Q \wedge R \wedge \neg S \wedge \bigcirc(\neg P \wedge \neg Q \wedge \neg S))))
\end{aligned}
$$

The entire process for computing and filtering the boundary conditions takes 0.43 seconds. Boundary conditions $1-3$ are computed for the safety case, while the last condition is for the liveness goal. When we compare the pattern-based derived boundary condition $\diamond(P \wedge R)$ with those computed with our technique, we observe that the former implies our boundary condition 1 (but not vice versa). That is, boundary condition 1 is more general than that derived by the pattern. The mentioned implication has been verified using a simple LTL satisfiability check ( $f_{1}$ implies $f_{2}$ iff $f_{1} \wedge \neg f_{2}$ is unsatisfiable). From the point of view of succinctness, clearly our boundary conditions, computed from loop-free path conditions in the tableau, are more complex and less readable than that derived by the pattern.

When applying our technique to the Retraction1 pattern, the approach computes the following potential boundary conditions, where 1 and 2 correspond to the safety case, and 3 to the liveness case:

$$
\begin{aligned}
& \text { 1. } \diamond(\neg P \wedge Q) \quad \text { 2. } \diamond(P \wedge \neg Q \wedge \bigcirc(\neg P \wedge Q)) \\
& \text { 3. } \diamond(P \wedge \square((\neg P \vee \neg Q) \vee(P \wedge \neg Q \wedge \bigcirc(\neg P \vee \neg Q))))
\end{aligned}
$$

The first two are discarded because they do not satisfy the minimality condition, while formula 3 is identified as a divergence. The whole boundary condition computation takes just 0.28 seconds. Again, our computed boundary condition is not as succinct as the pattern-based one. And also as in the previous pattern, our computed boundary condition is more general than the pattern-based derived one (the pattern based boundary condition implies formula 3 ).

When applying our technique to the Retraction2 pattern, the tool computes the following potential boundary condition, which is a divergence:

$$
\text { 1. } \diamond((\neg P \wedge Q \wedge \neg R) \vee(P \wedge(\neg Q \wedge \neg S \vee Q \wedge \neg R)))
$$

The whole divergence computation takes 0.48 seconds. Since this pattern does not consider liveness goals, the boundary conditions were computed for the safety case only. As with the previous patterns, formula 1 is implied by that derived by the Retraction 2 pattern, and consequently, our approach is again more general.

Notice that for the Achieve-Avoid pattern our approach is able to produce boundary conditions that are not identified by the pattern. Thus, these characterise additional divergent cases, that can be very useful to engineers when analysing conflicting situations in goal specifications.

\subsection{An example not captured by patterns}

Consider the TCP network protocol, which provides reliable in-order delivery of packets in packet based data transmission. For simplification, let us assume that the protocol can send one packet at a time, i.e., it waits for an acknowledgement (ack) before sending the next packet. Briefly, the following liveness goals are elicited for this protocol:

Goals: Achieve[DeliveredWhenSent]

FormalDef: $\square($ send $\rightarrow(\neg$ ack $\mathcal{U}$ delivered $))$

Goals: Achieve[WaitACKBeforeSendAgain]

FormalDef: $\square($ delivered $\rightarrow(\neg$ send $\mathcal{U}$ ack $))$

Notice that this example cannot be matched to any of the above patterns. Our technique is able to analyse this specification, and computes, in 1.31 seconds, the following divergences:

$$
\begin{aligned}
\text { 1. } \diamond(\text { send } \wedge \square((\neg \text { ack } \wedge(\neg \text { delivered } \vee \text { send }) \vee \text { ack } \wedge \neg \text { delivered }) \\
\vee(\neg \text { ack } \wedge \neg \text { delivered } \wedge \text { send } \wedge \bigcirc(\neg \text { ack } \wedge(\neg \text { delivered } \vee \text { send }) \\
\vee(\text { ack } \wedge \neg \text { delivered }))))
\end{aligned}
$$

The first boundary condition evidences a divergence if, from a certain point onwards, after sending a packet, either the ack signal is never received, or it is received before the packet has been delivered. The second boundary condition indicates that the goals are divergent when a packet was delivered, but its corresponding ack is never received, or a new packet is sent before receiving the ack.

\subsection{Discussion}

Let us briefly discuss about the scalability of our approach and the readability of our computed boundary conditions. Table ?? summarises for each case study the size of the constructed tableau (number of nodes and transitions), the number of computed potential boundary conditions (pBCs) and the resulting divergences (BCs), the analysis time required for constructing the tableau and for the entire process (in seconds). Moreover, to assess the readability of the BCs computed, we evaluate the succinctness of the boundary condition computed for each case study (we choose the biggest one, when multiple pBCs are computed). We measure the number of literals (Lit.), and the number of logical and temporal operators involved in the formula (L.Op. and T.Op., 
resp.). We also consider additional, more complex, specifications, namely, the ATM [?], Telephone [?], and London Ambulance Service (LAS) [?] (3, 5 and 5 formulas, resp.).

Table 1: Scalability and Succinctness Summary

\begin{tabular}{|c|c|c|c|c|c|c|c|}
\hline Case & Tableau & pBCs & \multicolumn{2}{|c|}{ BC Succinctness } & Tableau & Total \\
\cline { 5 - 6 } Study & nodes/trans & /BCs & Bit. & L.Op. & T.Op. & Time & Time \\
\hline Elevator & $24 / 48$ & $2 / 1$ & 6 & 8 & 3 & 0.64 & 0.80 \\
\hline RRCS & $53 / 120$ & $5 / 1$ & 9 & 10 & 1 & 0.31 & 0.50 \\
\hline TCP & $34 / 86$ & $2 / 2$ & 14 & 20 & 3 & 0.92 & 1.31 \\
\hline ATM & $72 / 206$ & $4 / 3$ & 13 & 16 & 3 & 2.08 & 2.71 \\
\hline Telephone & $163 / 508$ & $4 / 1$ & 13 & 19 & 2 & 8.84 & 11.43 \\
\hline LAS & $93 / 184$ & $1 / 1$ & 42 & 58 & 1 & 11.58 & 11.68 \\
\hline Achieve-Avoid & $34 / 86$ & $4 / 4$ & 17 & 28 & 4 & 0.27 & 0.43 \\
\hline Retraction1 & $12 / 24$ & $3 / 1$ & 7 & 11 & 3 & 0.02 & 0.20 \\
\hline Reatraction2 & $30 / 66$ & $1 / 1$ & 8 & 12 & 1 & 0.45 & 0.48 \\
\hline
\end{tabular}

Table ?? shows that almost all the analysis time is spent in constructing the tableau. We observe that this is more evident in the specifications that contain more liveness goals, as the TCP, ATM and Telephone case studies. This is due to the fact that removing the nodes that do not satisfy eventualities requires multiple visits to the tableau. Although we need to perform a more thorough scalability evaluation, the efficiency of our approach is promising, at least when analysing specifications that involve only safety goals, or a restricted number of liveness goals.

Table ?? also shows that the computed boundary conditions involve a considerable number of literals, logical and temporal operators, hindering their readability. This is, evidently, one of the most important issues we need to overcome to help the engineer in understanding the divergences computed and resolving the goal models.

\section{RELATED WORK}

Various informal and semi-formal approaches have been proposed for detecting conflicts in requirements models [?, ?, ?]. In addition, formal methods for detecting inconsistency have also been proposed in [?, ?, ?, ?, ?, ?]. These approaches focus on logical inconsistencies only, or ontology mismatch. Other techniques for reasoning about inconsistencies based on abduction such as [?] consider generating explanations (in the form of conjunctions of ground literals) for strong inconsistencies in requirements expressed in quasi-classical logic. Our approach focuses on detecting weak inconsistencies and automatically generating general LTL expressions that characterise situations in which inconsistencies may arise. Techniques such as [?], perform consistency checks for requirements expressed as conditional scenarios as a precursor for model synthesis. These consistency checks would identify inconsistency between two enabled charts. Our work on the other hand focuses on finding a characterisation for enabling conditions that could lead to an inconsistency. Conflicts in non-functional requirements have also been considered in [?, ?, ?]. For instance, [?] proposes a catalogue of conflicts, categorising these (pairwise) according to the frequency in which they occur together.

In [?], an approach for detecting conflicts between feature descriptions expressible in LTL is presented. It focuses on strong conflicts (i.e., mutually inconsistent features), applies to features of the form $p \rightarrow q \mathcal{U}(r \vee d)$, and uses a model checker for the conflict check. Our approach focuses on identifying weak conflicts, characterising them by boundary conditions, and is applicable to a wider class of LTL formulas.
The technique presented in [?] combines model checking and machine learning to automatically generate a set of obstacle conditions with respect to goals and domain properties expressed in LTL. It supposes however that each goal is satisfiable within its domain, and focuses on identifying the conditions under which the goal and domain may be inconsistent. It does not handle situations that arise because the goals themselves are inconsistent with one another.

The resolution of conflicts has been the subject of recent work, e.g., [?, ?]. The technique proposed in [?] makes use of argumentation patterns to elicit, compose and relate stakeholders beliefs. The technique assumes conflicts have already been elicited, and calculates an inconsistency score between beliefs and goals (described informally). These scores are then used as a guide for engineers as to which goals to resolve. Approaches like [?] propose methods that generate consistency specifications by construction, eliminating the need for detection. The problem of detecting inconsistency in specifications is related to that of vacuity detection [?, ?]. The latter may be a result of the former though inconsistency detection cannot be reduced to a vacuity check. It is also somewhat related to that of detecting overconstrained specifications. For instance, [?] proposes an algorithm for extracting a core set of assertions that cause an inconsistency in an Alloy model. The method assumes that inconsistency is already known to exist. Our method however attempts to find the assertion (i.e., $B C$ ) that would lead to the inconsistency. It also relates to that of realisability of specifications, e.g., $[?, ?]$, in that conflicting goals may lead to unrealisable requirements.

\section{CONCLUSION AND FUTURE WORK}

Detecting inconsistencies early on in the requirements engineering process helps avoiding costly software repairs, and also supports systematic requirements' elicitation and verification activities. This paper presents a novel approach for detecting divergences in goal specifications, and generating boundary conditions that characterise the cases under which a strong inconsistency could potentially arise. To the best of our knowledge, our approach is the first fully automated technique for computing goal conflicts that applies both to safety and to a wide range of liveness goals, specified in LTL. Our tableau-based method is guaranteed to produce correct results and to terminate. Our approach computes more general formulas than previous, related techniques. However, as our boundary conditions are automatically produced from tableau paths that lead to inconsistencies, they are often longer and less comprehensible in some cases than the more compact ones derived from patterns.

The presented work opens various lines for future work. For instance, a source of incompleteness of our approach is related to the way we interpret divergent tableau paths, applying a kind of generalisation through the application of temporal operators. We are studying alternative, stronger ways of dealing with these divergent paths, to be able to identify more boundary conditions. We are also considering the use of inductive learning techniques to support the inference of more generalised expressions.

Our approach relies on a tableaux construction; we plan to study more efficient tableau procedures, e.g., [?], to increase our technique's efficiency. We also plan to exploit tableaux structures for other problems related to requirements specifications, such as obstacle condition identification [?]. 